\title{
Phenotypic and Genotypic Characterization of Some Thermophilic Species of Bacillus
}

\author{
By R. J. SHARP,* K. J. BOWN AND A. ATKINSON \\ Public Health Laboratory Service, Centre for Applied Microbiology and Research, \\ Porton Down, Salisbury, Wiltshire SP4 0JG
}

(Received 19 March 1979; revised 13 August 1979)

\begin{abstract}
Some thermophilic species of Bacillus were characterized using biochemical tests, antibiotic sensitivity, bacteriocin and bacteriophage sensitivity, esterase patterns, DNA hybridization and $\% \mathrm{G}+\mathrm{C}$ content. The three caldo-active strains of bacilli isolated by Heinen (1971) were compared with strains of $B$. stearothermophilus. Eight of the strains examined showed characteristics which enabled them to be placed in the three main taxonomic groups suggested by Walker \& Wolf (1971). Two strains showed characteristics of both groups 1 and 3. Examination of genotype data showed taxonomic groupings which differed from those based on phenotypic characterization.
\end{abstract}

\section{INTRODUCTION}

Over the past decade there has been a considerable increase in interest in the thermophilic bacteria and their enzymes (Brock \& Freeze, 1969; Atkinson, 1973; Singleton \& Amelunxen, 1973; Jung et al., 1974; Williams, 1975; Atkinson et al., 1975). We have examined the taxonomic position and relationships between strains of Bacillus stearothermophilus, $B$. coagulans, the caldo-active bacteria isolated by Heinen (1971) and the thermophile isolated by Epstein \& Grossowicz (1969). The latter was at first identified as a subspecies of $B$. coagulans but later as a starch-negative strain of B. stearothermophilus (Cazzulo et al., 1969, 1970; Atkinson et al., 1975). Three other thermophilic bacilli isolated in this laboratory were also examined.

\section{METHODS}

Strains and growth. Strains of Bacillus stearothermophilus used were: ATCC 12016; NCA 1503 (NCIB 8924); strain 10, from Dr N. Welker, North Western University, Ill., U.S.A.; strain RS93, isolated from the hot water supply to the fermentation plant at the Microbiological Research Establishment, Porton, Salisbury (Sharp et al., 1979); strain 240, isolated from chalky soil in the Salisbury area; strain 262, isolated from an Icelandic hot water spring; and strain 136, isolated by Epstein \& Grossowicz (1969). Cultures of B. caldotenax, $B$. caldovelox and $B$. caldolyticus were from Dr W. Heinen, University of Nijmegen, Holland. Bacillus coagulans ATCC 8038, B. coagulans ATCC 12245, B. subtilis 168 (Trp $\left.{ }^{-}\right)$, B. amyloliquefaciens H1, B. megaterium KM and Escherichia coli $\mathrm{K} 12$ (Hfr) were also used.

All strains were normally stored as lyophilized cultures and routinely grown on Tryptone Soya Agar (TSBA) (Oxoid CM131). The liquid media used were as described by Sargeant et al. (1971).

Bacteriophages. Bacteriophages TP1C and TP84 were from Dr N. Welker. Bacteriophage ATCC 12016b was also used. Bacteriophages JS017, JS020 and JS025 were isolated from compost samples taken from the Salisbury area.

Biochemical tests. Sugars in basal peptone water were inoculated from a $6 \mathrm{~h}$ peptone water broth culture derived from an overnight plate culture. Citrate utilization was tested on Simmon's citrate slopes. Gelatin liquefaction, starch hydrolysis, casein hydrolysis, nitrate and nitrite reduction, indole production, acetoin production, growth in $0.02 \% \mathrm{NaN}_{3}$, catalase production and oxidase reaction were all examined by the 
methods of Cowan \& Steel (1974). Tolerance to saline was determined by inoculating bottles containing $10 \mathrm{ml}$ nutrient broth no. 2 (Oxoid CM67) supplemented with 1 to $10 \%$ (w/v) NaCl. Sabouraud dextrose agar plates were based on Oxoid agar (CM41). Readings were made at 1,2 and $3 \mathrm{~d}$.

Antibiotic sensitivity. Cultures were grown in peptone water broth for $6 \mathrm{~h}$. Disposable bioassay plates were prepared with $200 \mathrm{ml}$ Oxoid Sensitest agar (CM409) with agar at $3 \%(\mathrm{w} / \mathrm{v})$. The plates were seeded with $3 \mathrm{ml}$ culture, and allowed to dry. Oxoid antibiotic sensitivity discs (15) were then placed on them and the plates were incubated overnight at $60^{\circ} \mathrm{C}$. The zone width from the edge of the disc to the edge of the bacterial growth was then measured.

Temperature range for growth. Cultures were streaked on to plates of TSBA and incubated at a range of temperatures: above $50^{\circ} \mathrm{C}$, plates were incubated in closed polythene bags; above $65^{\circ} \mathrm{C}$, glass Petri dishes were used. The plates were examined daily up to $3 \mathrm{~d}$.

Bacteriocin sensitivity. A crude preparation of thermocin 93 (Sharp et al., 1979) was used. Growth inhibition was observed on agar plates using the well diffusion method.

Bacteriophage sensitivity. Phages were harvested after growth on $B$. caldotenax and titres were adjusted to $10^{6}$ plaque forming units (p.f.u.) $\mathrm{ml}^{-1}$. Dilutions of the bacteriophage were mixed with $0.1 \mathrm{ml}$ of a $6 \mathrm{~h}$ culture of the bacteria in $2 \mathrm{ml}$ of Trypticase (BBL) soft agar $\left(0.6 \%\right.$, w/v) containing $0.015 \mathrm{M}-\mathrm{CaCl}_{2}$ at $50^{\circ} \mathrm{C}$. After mixing, the soft agar was poured over the surface of TSBA plates and incubated at $60{ }^{\circ} \mathrm{C}$ overnight.

Constitutive enzyme production using API-ZYM test strips. The API-ZYM system (API, Invincible Rd, Farnborough) is a semi-quantitative micro-system designed for the study of enzyme activities. Bacteria were grown for $16 \mathrm{~h}$ on TSBA plates at $60^{\circ} \mathrm{C}$. Suspensions of cells were prepared in distilled water to give an absorbance of 1.5 at $450 \mathrm{~nm}$, and $60 \mu \mathrm{l}$ was added to each reaction cupule. The strips were incubated at $60^{\circ} \mathrm{C}$ for $10 \mathrm{~h}$ before reading the results.

Esterase electrophoresis. Cultures were grown overnight at $60^{\circ} \mathrm{C}$ in the medium of Sargeant $e t$ al. (1971). Cells were harvested by centrifuging at $10000 \mathrm{~g}$ for $30 \mathrm{~min}$ at $4{ }^{\circ} \mathrm{C}$, washed twice in $0.15 \mathrm{M}-\mathrm{NaCl}$ and finally suspended in $0.15 \mathrm{M}-\mathrm{NaCl}$ to give $3 \mathrm{~g}$ wet wt cells per $15 \mathrm{ml}$ saline. The suspensions were disrupted ultrasonically $(20 \mathrm{kHz}, 5 \times 1 \mathrm{~min})$ on ice and cell debris was removed by centrifugation at $48000 \mathrm{~g}$ for $1 \mathrm{~h}$ at $4{ }^{\circ} \mathrm{C}$. Cell extracts were concentrated 25 -fold in a Minicon B15 concentrator (Amicon) and stored at $-20^{\circ} \mathrm{C}$.

Gels were prepared by dissolving $23 \mathrm{~g}$ soluble starch in $200 \mathrm{ml}$ electrophoresis buffer ( $90 \mathrm{~mm}$-Tris, $90 \mathrm{~mm}$ boric acid, 3 mM-EDTA, pH 8.0) and heated with continuous stirring until maximum viscosity was attained. The solution was then poured on to plates $(190 \times 140 \mathrm{~mm})$ to give a $4 \mathrm{~mm}$ layer. Graphite electrodes were inserted $150 \mathrm{~mm}$ apart and wells $(9 \times 1 \mathrm{~mm})$ were cut $6 \mathrm{~mm}$ apart. The plates were stored overnight at $4{ }^{\circ} \mathrm{C}$ covered with Clingfilm.

Bromophenol blue was added as a marker to each extract prior to electrophoresis. Samples of $100 \mu \mathrm{l}$ were loaded and electrophoresis was carried out at $120 \mathrm{~V}$ for $2.5 \mathrm{~h}$ at ambient temperature. Electrophoresis buffer was dropped on to the gel surface intermittently to prevent excess heating or drying out.

The gels were stained as described by Baillie \& Norris (1963) and $R_{F}$ values for the esterase bands relative to the bromophenol blue front were determined.

Preparation and analysis of DNA. $\left[{ }^{32}\right.$ P]DNA was prepared from B. stearothermophilus NCA 1503 and B. caldotenax grown at $60^{\circ} \mathrm{C}$ and from B. subtilis 168 and B. coagulans ATCC 8038 grown at $35^{\circ} \mathrm{C}$ in the following medium $\left(\mathrm{g}^{-1}\right.$ ): Tris, 6 ; glucose, $10 ; \mathrm{KCl}, 5 ; \mathrm{MgSO}_{4} .7 \mathrm{H}_{2} \mathrm{O}, 1 ; \mathrm{NaCl}$, 4; glutamic acid, 5; Difco Casamino acids, 1; Difco yeast extract, $0 \cdot 1$; $\mathrm{L}$-tryptophan, $0 \cdot 025$; plus ${ }^{32} \mathrm{PO}_{4}{ }^{3-}\left[1 \cdot 2 \mathrm{mCi}^{-1}\left(44 \mathrm{MBq}^{-1}\right)\right]$. The $\mathrm{pH}$ was adjusted to $7 \cdot 4$ with $\mathrm{HCl}$. For unlabelled DNA, all strains were grown at $60^{\circ} \mathrm{C}$ in the medium of Sargeant et al. (1971) but with $1 \%(\mathrm{w} / \mathrm{v})$ glucose replacing sucrose as the major carbon source. Bacillus subtilis $168\left(\right.$ (Trp $\left.^{-}\right)$, B. amyloliquefaciens $\mathrm{H} 1$, B. megaterium KM, B. coagulans ATCC 8038 and ATCC 12445 and $E$. coli $\mathrm{K} 12 \mathrm{HfrH}$ were grown at $35^{\circ} \mathrm{C}$ in this medium.

Cells washed with $0.15 \mathrm{M}-\mathrm{NaCl}, 0.01 \mathrm{M}-\mathrm{Na}_{2} \mathrm{EDTA}, 0.01 \mathrm{M}-\mathrm{Tris} / \mathrm{HCl}(\mathrm{pH} 8.0)$ were treated with lysozyme

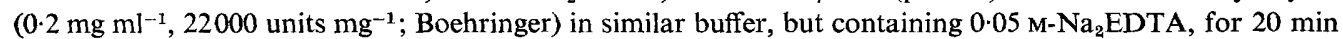
at $25^{\circ} \mathrm{C}$ and lysed with sodium dodecyl sulphate $\left(20 \mathrm{~g} \mathrm{l}^{-1}\right)$ at $70^{\circ} \mathrm{C}$ for $15 \mathrm{~min}$. The lysed material was incubated with Proteinase $\mathrm{K}\left(50 \mu \mathrm{g} \mathrm{ml}^{-1}, 20\right.$ units $\mathrm{mg}^{-1}$; Boehringer) for $16 \mathrm{~h}$ at ambient temperature. DNA was isolated as described by Marmur (1961) and further purified as described by Shah et al. (1976).

Thermal denaturation of purified DNA was carried out as described by Shah et al. (1976) in $0 \cdot 15 \mathrm{M}-\mathrm{NaCl}$, 0.015 M-sodium citrate, pH 7.0 (SSC) (Marmur \& Doty, 1962).

DNA-DNA hybridization was carried out essentially as described by Denhardt (1966) on Millipore HADP $25 \mathrm{~mm}$ diam. filters. Filters preloaded with $25 \mu \mathrm{g}$ sonicated, heat-denatured $\left(10{ }^{\circ} \mathrm{C}, 15 \mathrm{~min}\right)$ unlabelled DNA were annealed in $6 \times \mathrm{SSC}$ with $0 \cdot 2 \mu \mathrm{g}$ sonicated, heat-denatured $\left[{ }^{32} \mathrm{P}\right] \mathrm{DNA}$ at $65^{\circ} \mathrm{C}$ for $16 \mathrm{~h}$ under paraffin oil. Filters were then washed with $2 \times$ SSC, dried and counted. DNA from E. coli K12 HfrH was used as a standard for thermal denaturation and as a blank in hybridization experiments. 
Table 1. Sugar fermentations and biochemical reactions of Bacillus species

All results were recorded after $48 \mathrm{~h}$; further incubation did not result in any additional activity. $\mathrm{w}$ denotes weak positive reactions.

Sugar fermentations:

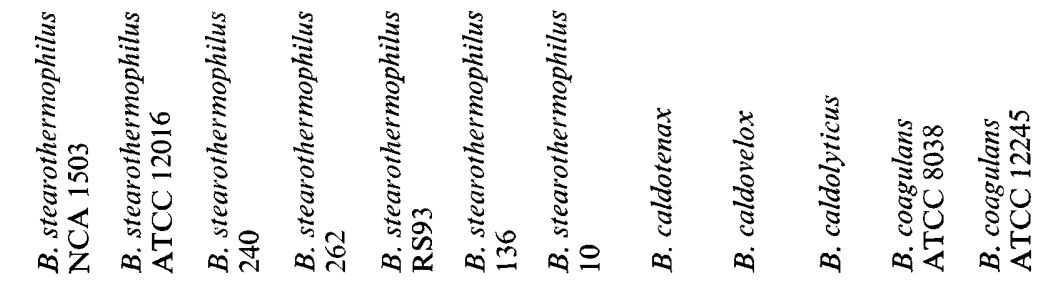

$\begin{array}{lllllllllllll}\text { Adonitol } & - & - & - & - & + & - & - & - & - & - & - & - \\ \text { Arabinose } & - & + & - & - & - & - & \mathrm{w} & - & - & - & - & - \\ \text { Dextrin } & + & - & + & + & - & + & + & + & + & + & + & + \\ \text { Erythritol } & - & - & - & - & - & + & - & - & - & - & - & - \\ \text { Galactose } & \mathrm{w} & + & - & - & - & - & \mathrm{w} & - & - & - & + & + \\ \text { Glucose } & + & + & + & + & \mathrm{w} & + & + & + & + & + & + & + \\ \text { Glycerol } & + & + & + & - & + & \mathrm{w} & + & + & + & \mathrm{w} & + & \mathrm{w} \\ \text { Glycogen } & + & \mathrm{w} & + & + & - & - & + & - & - & + & \mathrm{w} & - \\ \text { Inositol } & - & - & - & - & \mathrm{w} & \mathrm{w} & - & - & - & - & - & - \\ \text { Lactose } & - & - & - & - & - & - & \mathrm{w} & - & - & - & + & + \\ \text { Laevulose } & + & \mathrm{w} & + & - & \mathrm{w} & + & + & + & \mathrm{w} & \mathrm{w} & + & + \\ \text { Maltose } & + & + & + & + & - & + & + & + & + & \mathrm{w} & + & + \\ \text { Mannitol } & - & \mathrm{w} & - & - & + & + & - & \mathrm{w} & + & \mathrm{w} & \mathrm{w} & - \\ \text { Mannose } & + & + & + & \mathrm{w} & \mathrm{w} & - & + & + & + & + & + & + \\ \text { Raffinose } & + & - & - & - & - & - & + & \mathrm{w} & \mathrm{w} & - & - & - \\ \text { Rhamnose } & - & - & - & - & - & \mathrm{w} & - & - & - & - & + & - \\ \text { Salicin } & - & \mathrm{w} & - & - & - & \mathrm{w} & - & - & - & - & + & + \\ \text { Starch } & + & - & + & \mathrm{w} & - & - & + & - & - & + & \mathrm{w} & \mathrm{w} \\ \text { Sucrose } & + & - & + & + & + & + & + & + & + & + & - & + \\ \text { Sorbitol } & - & - & - & - & \mathrm{w} & + & - & - & - & - & + & - \\ \text { Trehalose } & + & - & + & - & \mathrm{w} & \mathrm{w} & + & - & + & + & + & + \\ \text { Xylose } & - & + & - & - & - & - & + & - & - & - & \mathrm{w} & \mathrm{w}\end{array}$

Biochemical reactions:

$\begin{array}{lllllllllllll}\mathrm{NaCl} \text { tolerance }(\%) & 3 & 1 & 3 & 4 & 5 & 7 & 3 & 3 & 2 & 2 & 2 & 2\end{array}$

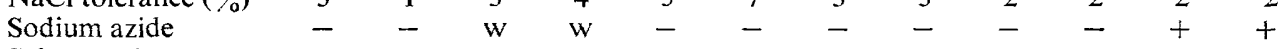

Sabouraud agar $\quad-\quad-\quad-\quad-\quad-\quad-\quad-\quad-\quad-$

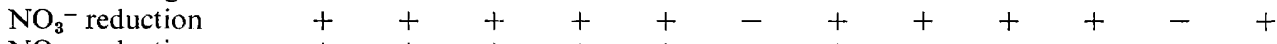

$\mathrm{NO}_{2}^{-}$-reduction $+\quad+\quad+\quad+\quad+\quad-\quad+\quad-\quad-\quad-\quad-$

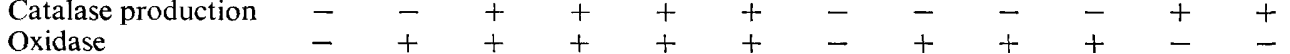

Gelatin hydrolysis $-\mathrm{w}-\mathrm{w}+\mathrm{w}+\mathrm{w}+\mathrm{t}+$

Casein digestion $\mathrm{w} \quad \mathrm{w}+\mathrm{w}_{-}+\mathrm{w}_{+}+\mathrm{w}_{-} \mathrm{w}$

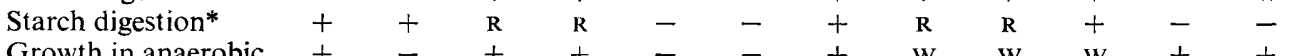

dextrose broth

Anaerobic reduction of $\mathrm{NO}_{3}^{-}$to gas

No strain utilized aesculin, dulcitol, inulin or citrate; no strain produced indole or acetoin.

* +, Large zone of hydrolysis; $\mathrm{R}$, restricted zone of hydrolysis.

\section{RESULTS AND DISCUSSION}

The main taxonomic characters which distinguished B. coagulans ATCC 8038 and ATCC 12245 from the other 10 thermophiles tested agreed with those recorded by Buchanan \& Gibbons (1974). The B. coagulans strains grew readily on Sabouraud dextrose agar and in sodium azide broth (Table 1). Their maximum and minimum growth temperatures were $61{ }^{\circ} \mathrm{C}$ and $25^{\circ} \mathrm{C}$, respectively (Table 5). They also showed a greater overall sensitivity to 


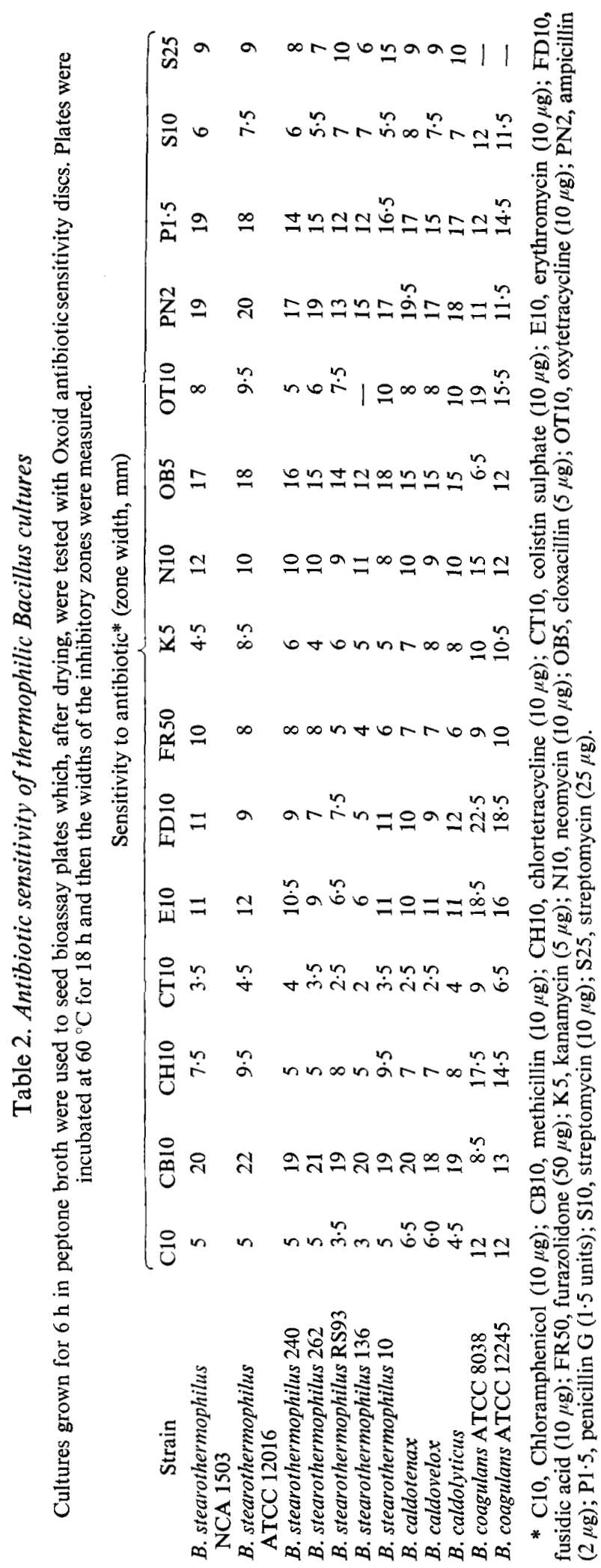


Table 3. Phage and bacteriocin sensitivity of thermophilic Bacillus cultures

Bacteriophage suspensions $\left(10^{6}\right.$ p.f.u. $\left.\mathrm{ml}^{-1}\right)$ and dilutions were plated with $0 \cdot 1 \mathrm{ml}$ of $6 \mathrm{~h}$ bacterial cultures and incubated at $60^{\circ} \mathrm{C}$ for $18 \mathrm{~h}$. Thermocin 93 was added to wells cut into TSBA plates seeded with bacterial suspensions; plates were incubated at $60^{\circ} \mathrm{C}$ for $18 \mathrm{~h}$ and then zone diameters were recorded.

\begin{tabular}{|c|c|c|c|c|c|c|c|}
\hline \multirow[b]{2}{*}{ Strain } & \multicolumn{6}{|c|}{ Efficiency of plating of phage* } & \multirow{2}{*}{$\begin{array}{c}\text { Thermocin } \\
93 \text { (zone } \\
\text { diam., } \\
\text { mm) }\end{array}$} \\
\hline & JS017 & JS020 & JS025 & TP1C & TP84 & $12016 b$ & \\
\hline $\begin{array}{l}\text { B. stearothermophilus } \\
\text { NCA } 1503\end{array}$ & - & - & - & + & ++ & - & 22 \\
\hline $\begin{array}{l}\text { B. stearothermophilus } \\
\text { ATCC } 12016\end{array}$ & +++ & - & - & - & +++ & $+t+t$ & 20 \\
\hline B. stearothermophilus 240 & - & - & - & - & +++ & $+t+t$ & 18 \\
\hline B. stearothermophilus 262 & ++ & - & - & + & ++ & + & 21 \\
\hline B. stearothermophilus RS93 & $++t+$ & +++ & $+t+t$ & + & + & - & 0 \\
\hline B. stearothermophilus 136 & - & - & - & - & - & - & 0 \\
\hline B. stearothermophilus 10 & ++ & - & - & + & ++ & - & 24 \\
\hline B. caldotenax & +++ & ++++ & +++ & $+++t$ & ++++ & ++++ & 22 \\
\hline B. caldovelox & $++t$ & ++++ & +++ & ++++ & +++ & $+t+t$ & 22 \\
\hline B. caldolyticus & +++ & +++ & +++ & +++ & ++ & ++ & 22 \\
\hline B. coagulans ATCC 8038 & - & - & - & - & - & - & 0 \\
\hline B. coagulans ATCC 12245 & - & - & - & - & - & - & 0 \\
\hline
\end{tabular}

$*++++$, Plating efficiency of 1 to $0 \cdot 1 ;+++$, plating efficiency of $0 \cdot 1$ to $0 \cdot 01 ;++$, plating efficiency of 0.01 to 0.0001 ; + , indication of low level phage infection; - , no visible indication of phage infection.

\section{Table 4. Constitutive enzyme production by thermophiles detected using the API-ZYM system}

Bacteria were grown for $16 \mathrm{~h}$ at $60^{\circ} \mathrm{C}$ on TSBA. Cells were suspended in distilled water $\left(A_{450}=1 \cdot 5\right)$ and $60 \mu \mathrm{l}$ was added to each reaction cupule. The strips were incubated at $60^{\circ} \mathrm{C}$ for $10 \mathrm{~h}$ before reading the results. Enzyme production was assessed qualitatively on a scale 0 to 5, 0 indicating no enzyme production, and 5 high enzyme production.

Enzyme detected in reaction cupule*

\section{Strain}

B. stearothermophilus NCA 1503

B. stearothermophilus ATCC 12016

B. stearothermophilus 240

B. stearothermophilus 262

B. stearothermophilus RS93

B. stearothermophilus 136

B. stearothermophilus 10

B. caldotenax

B. caldovelox

B. caldolyticus

\begin{tabular}{llllllllllllllllllllll}
\multicolumn{11}{c}{ Enzyme detected in reaction cupule* } \\
\hline 1 & 2 & 3 & 4 & 5 & 6 & 7 & 8 & 9 & 10 & 11 & 12 & 13 & 14 & 15 & 16 & 17 & 18 & 19 & 20 \\
0 & 4 & 3 & 4 & 3 & 2 & 1 & 1 & 2 & 2 & 2 & 4 & 4 & 2 & 2 & 1 & 0 & 0 & 0 & 0 \\
0 & 2 & 2 & 3 & 2 & 1 & 0 & 0 & 1 & 0 & 2 & 4 & 4 & 3 & 2 & 2 & 1 & 0 & 0 & 0 \\
0 & 2 & 2 & 3 & 2 & 2 & 1 & 1 & 1 & 4 & 2 & 2 & 0 & 1 & 1 & 3 & 2 & 1 & 0 & 0 \\
0 & 1 & 1 & 1 & 1 & 1 & 0 & 0 & 0 & 0 & 1 & 2 & 0 & 0 & 2 & 2 & 1 & 1 & 0 & 0 \\
0 & 1 & 1 & 1 & 1 & 0 & 0 & 0 & 0 & 0 & 2 & 3 & 0 & 0 & 0 & 1 & 0 & 0 & 0 & 0 \\
0 & 2 & 2 & 2 & 1 & 1 & 2 & 1 & 1 & 3 & 3 & 2 & 0 & 0 & 0 & 1 & 1 & 0 & 0 & 0 \\
0 & 1 & 2 & 2 & 1 & 0 & 0 & 0 & 0 & 0 & 3 & 2 & 3 & 1 & 2 & 3 & 1 & 0 & 0 & 0 \\
0 & 5 & 3 & 4 & 3 & 3 & 3 & 2 & 3 & 3 & 4 & 4 & 4 & 1 & 1 & 4 & 3 & 1 & 0 & 0 \\
0 & 5 & 4 & 4 & 3 & 2 & 2 & 1 & 2 & 2 & 4 & 4 & 4 & 2 & 3 & 4 & 4 & 2 & 1 & 0 \\
0 & 4 & 3 & 3 & 3 & 2 & 1 & 1 & 2 & 2 & 3 & 3 & 3 & 2 & 3 & 3 & 4 & 1 & 1 & 0
\end{tabular}

* (1) Negative control, (2) alkaline phosphatase, (3) esterase (C4), (4) esterase lipase (C8), (5) lipase (C14), (6) leucine arylamidase, (7) valine arylamidase, (8) cystine arylamidase, (9) trypsin, (10) chymotrypsin, (11) acid phosphatase, (12) phosphoamidase, (13) $\alpha$-galactosidase, (14) $\beta$-galactosidase, (15) $\beta$-glucuronidase, (16) $\alpha$-glucosidase, (17) $\beta$-glucosidase, (18) $N$-acetyl- $\beta$-glucosaminidase, (19) $\alpha$-mannosidase, (20) $\alpha$-fucosidase.

antibiotics (Table 2) but were not inhibited by thermocin 93 (Table 3). DNA hybridization results showed a low percentage of hybridization with DNA from $B$. stearothermophilus NCA 1503 and B. caldotenax and their DNA $\% \mathrm{G}+\mathrm{C}$ contents were lower than for the other strains examined, falling in the range 47 to $48 \%$ (Table 7).

The three caldo-active strains of Heinen (1971) and the other seven thermophiles could be placed into the three major groups of Walker \& Wolf (1971) (Table 8). In group $1 B$. stearothermophilus ATCC 12016 complies with their three main criteria; B. caldotenax and $B$. caldovelox also showed restricted hydrolytic activity on starch, grew poorly in anaerobic 
Table 5. Growth temperature of thermophilic Bacillus cultures

Strains were examined for growth on TSBA following overnight incubation, unless indicated otherwise.

Growth temperature $\left({ }^{\circ} \mathrm{C}\right)$

\begin{tabular}{lccc}
\multicolumn{1}{c}{ Strain } & Maximum & Minimum & Optimum \\
B. stearothermophilus NCA 1503 & 74 & 41 & 60 to 65 \\
B. stearothermophilus ATCC 12016 & 76 & 40 & 60 to 65 \\
B. stearothermophilus 240 & 77 & 41 & 60 to 70 \\
B. stearothermophilus 262 & 78 & 42 & 60 to 70 \\
B. stearothermophilus RS93 & 68 & 39 & 60 to 65 \\
B. stearothermophilus 136 & 65 & 41 & 55 to 58 \\
B. stearothermophilus 10 & 75 & 41 & 60 to 65 \\
B. caldotenax & 85 & - & 80 \\
B. caldovelox & 76 & - & 60 to 70 \\
B. caldolyticus & 82 & - & 72 \\
B. coagulans ATCC 8038 & 61 & $25(3 \mathrm{~d})$ & 50 to 55 \\
B. coagulans ATCC 12245 & 61 & $25(3 \mathrm{~d})$ & 50 to 55
\end{tabular}

Table 6. Electrophoretic mobility of esterases in cell extracts of thermophilic Bacillus cultures

Conditions were as described in Methods. $R_{F}$ values were determined with respect to bromophenol blue.

$$
\text { Strain }
$$

B. stearothermophilus NCA 1503

B. stearothermophilus 10

B. stearothermophilus RS93

B. stearothermophilus 136

B. stearothermophilus 240

B. caldolyticus

B. stearothermophilus ATCC 12016

B. stearothermophilus 262

B. caldotenax

B. caldovelox

$$
\begin{array}{r}
R_{F} \text { value* } \\
0 \cdot 20(\mathrm{~s}) \\
0 \cdot 21(\mathrm{~s}) \\
0 \cdot 30(\mathrm{~s}) \\
0 \cdot 30(\mathrm{~s}) \\
0 \cdot 14(\mathrm{~s}) \\
\text { (i) } 0 \cdot 18(\mathrm{~s}) \\
\text { (ii) } 0 \cdot 30(\mathrm{~s}) \\
\text { (i) } 0 \cdot 13(\mathrm{w}) \\
\text { (ii) } 0 \cdot 30(\mathrm{~s}) \\
\text { (iii) } 0 \cdot 67(\mathrm{~s}) \\
\text { (i) } 0 \cdot 06(\mathrm{w}) \\
\text { (ii) } 0 \cdot 18(\mathrm{~s}) \\
\text { (iii) } 0 \cdot 37(\mathrm{~s}) \\
\text { (i) } 0 \cdot 18(\mathrm{~s}) \\
\text { (ii) } 0 \cdot 37(\mathrm{~s}) \\
\text { (i) } 0 \cdot 18(\mathrm{~s}) \\
\text { (ii) } 0.37(\mathrm{~s})
\end{array}
$$

* s, Strongly staining band; w, weakly staining band.

glucose broth but did not reduce nitrate to gas under anaerobic conditions. Walker \& Wolf (1971) also described three strains which failed to reduce nitrite to $N_{2}$ but which otherwise readily fall into group 1 . These three strains were placed into sub-group $1 b_{4}$ (Walker \& Wolf, 1971).

Bacillus stearothermophilus 136 and RS93 appeared to belong to group 2 since neither of them hydrolysed starch. Strains NCA 1503, 10 and 240 belonged to group 3; they displayed good growth in glucose broth anaerobically and strong hydrolytic action on starch. Bacillus caldolyticus hydrolysed starch but grew poorly in anaerobic dextrose broth; $B$. stearothermophilus 262 grew well in anaerobic dextrose broth but starch hydrolysis was restricted. These two thermophiles therefore appeared to have characters of both group 1 and group 3 organisms.

To obtain further evidence for the taxononic relationships between the 10 thermophiles, a comparison was made of their esterase enzymes. Baillie \& Walker (1968) showed a close relationship between characterization on biochemical tests and esterase patterns in 
Table 7. DNA homology and $\% G+C$ among mesophilic and thermophilic Bacillus cultures

Nitrocellulose filters ( $25 \mathrm{~mm}$ diam.) were loaded with $25 \mu \mathrm{g}$ total denatured DNA and reassociation was performed with $0.2 \mu \mathrm{g}$ sonicated, denatured [32P]DNA in $6 \times \mathrm{SSC}$ at $65^{\circ} \mathrm{C}$ for $16 \mathrm{~h}$ under paraffin oil. Reassociation values $(\alpha)$ were defined as:

$$
\alpha=100 \times \frac{\text { (Counts bound to heterologous filters) }- \text { (Counts bound to } E \text {. coli filters) }}{\text { (Counts bound to homologous filters) }- \text { (Counts bound to } E \text {. coli filters) }}
$$

DNA $\% \mathrm{G}+\mathrm{C}$ was calculated from $\mathrm{mol} \% \mathrm{G}+\mathrm{C}=2 \cdot 44\left(T_{m}-69 \cdot 3\right)$ (Marmur \& Doty, 1962) from melting curves determined in SSC.

Percentage of reassociation with [32P]DNA from:

\begin{tabular}{|c|c|c|c|c|c|}
\hline $\begin{array}{l}\text { Total unlabelled } \\
\text { DNA from: }\end{array}$ & $\mathrm{mol} \% \mathrm{G}+\mathrm{C}$ & $\begin{array}{l}\text { B. stearo- } \\
\text { thermophilus } \\
\text { NCA } 1503\end{array}$ & B. caldotenax & B. subtilis 168 & $\begin{array}{l}\text { B. coagulans } \\
\text { ATCC } 8038\end{array}$ \\
\hline $\begin{array}{l}\text { B. stearothermophilus } \\
\text { NCA } 1503\end{array}$ & $51 \cdot 9$ & 100 & 23 & 17 & 11 \\
\hline$B$. caldotenax & $64 \cdot 8$ & 22 & 100 & 9 & 9 \\
\hline B. subtilis $168\left(\operatorname{Trp}^{-}\right)$ & $44 \cdot 1$ & 19 & 11 & 100 & 10 \\
\hline B. coagulans ATCC 8038 & $48 \cdot 3$ & 9 & 7 & 10 & 100 \\
\hline B. stearothermophilus 10 & $50 \cdot 9$ & 85 & 19 & 16 & 12 \\
\hline $\begin{array}{l}\text { B. stearothermophilus } \\
240\end{array}$ & $52 \cdot 3$ & 92 & 21 & 14 & 15 \\
\hline $\begin{array}{l}\text { B. stearothermophilus } \\
\text { ATCC } 12016\end{array}$ & $54 \cdot 2$ & 74 & 25 & 8 & 16 \\
\hline $\begin{array}{l}\text { B. stearothermophilus } \\
\text { RS93 }\end{array}$ & $54 \cdot 5$ & 77 & 23 & 9 & 15 \\
\hline B. caldolyticus & $52 \cdot 3$ & 53 & 38 & 11 & 9 \\
\hline $\begin{array}{l}\text { B. stearothermophilus } \\
262\end{array}$ & $62 \cdot 2$ & 28 & 66 & 7 & 11 \\
\hline B. caldovelox & $65 \cdot 1$ & 23 & 88 & 5 & 8 \\
\hline $\begin{array}{l}\text { B. stearothermophilus } \\
136\end{array}$ & $47 \cdot 5$ & 12 & 11 & 10 & 66 \\
\hline $\begin{array}{l}\text { B. coagulans } \\
\text { ATCC } 12245\end{array}$ & $46 \cdot 9$ & 18 & 6 & 11 & 88 \\
\hline B. amyloliquefaciens $\mathrm{H} 1$ & $43 \cdot 2$ & 5 & 8 & 19 & 10 \\
\hline B. megaterium $\mathrm{KM}$ & $36 \cdot 7$ & 9 & 4 & 8 & 10 \\
\hline
\end{tabular}

B. stearothermophilus. The results in Table 6 confirm the subdivision into three groups (Table 9) corresponding closely to those of Walker \& Wolf (1971); group 1, however, showed some variation. The esterase enzyme pattern of $B$. stearothermophilus 262 closely resembled that of the caldo-active bacteria but had a third, weakly staining band. Bacillus stearothermophilus ATCC 12016 produced two strongly staining bands and one weakly staining band, but with $R_{F}$ values differing from those of other members of group 1.

Determination of the percentage similarity of the strains based on 43 characters (those in Table 1, thermocin 93 sensitivity, and sensitivity to phages TP1C, TP84 and 12016b) showed two groups with $>80 \%$ similarity (Table 11). Bacillus caldotenax and B. caldovelox showed $98 \%$ similarity, and B. caldolyticus showed $90 \%$ similarity with this group. Bacillus stearothermophilus NCA 1503 and 10 showed $91 \%$ similarity and $80 \%$ similarity with $B$. stearothermophilus 240 and with B. caldolyticus. Bacillus stearothermophilus 262 showed 70 to $80 \%$ similarity to both groups but had an $88 \%$ similarity to B. stearothermophilus 240 . Bacillus stearothermophilus 136 and RS93 showed $80 \%$ similarity with each other but less than $65 \%$ with the other groups, and appeared as a third group.

DNA hybridization results and \% $\mathrm{G}+\mathrm{C}$ content (Table 7) supported the presence of three main groups (Table 10). Bacillus stearothermophilus 136 was similar to both strains of $B$. coagulans. Bacillus caldotenax and B. caldovelox had similar $\% \mathrm{G}+\mathrm{C}$ contents and showed a high percentage of DNA hybridization. Bacillus stearothermophilus 262 had a $\% \mathrm{G}+\mathrm{C}$ 
Table 8. Classification of strains based on the system of Walker \& Wolf (1971)

Group 1

\author{
B. stearothermophilus ATCC 12016 \\ B. caldotenax \\ $B$. caldovelox \\ $B$. caldolyticus \\ B. stearothermophilus 262
}

Group 2

B. stearothermophilus RS93

B. stearothermophilus 136
Group 3

B. stearothermophilus NCA 1503

B. stearothermophilus 10

B. stearothermophilus 240

B. caldolyticus

B. stearothermophilus 262

\section{Table 9. Classification of strains based on the electrophoretic mobility of} their esterase enzymes

Group 1

Two or more bands

B. caldotenax

B. caldovelox

B. caldolyticus

B. stearothermophilus $262 *$

B. stearothermophilus ATCC $12016 \dagger$
Group 2

One band, similar $R_{F}$ values

$B$. stearothermophilus 136

B. stearothermophilus RS93
Group 3

One band, similar $R_{F}$ values

$B$. stearothermophilus NCA 1503

B. stearothermophilus 10

B. stearothermophilus 240

* This strain showed three bands: two were identical with those of the caldo-active bacteria, the third was a weakly stained band.

$\uparrow$ This strain showed three bands, two strongly stained and one weakly stained; they did not correspond to the bands produced by the caldo-active bacteria.

Table 10. Classification of strains based on DNA hybridization data and $\% G+C$ content

\section{Group 1}

B. caldotenax

B. caldovelox

B. stearothermophilus 262

\section{Group 2}

B. stearothermophilus 136

$B$. coagulans strains
Group 3

B. stearothermophilus NCA 1503

B. stearothermophilus 10

B. stearothermophilus 240

B. stearothermophilus ATCC 12016

B. stearothermophilus RS93

$B$. caldolyticus

content similar to those of $B$. caldotenax and $B$. caldovelox with a slightly lower percentage of DNA hybridization. Bacillus stearothermophilus strains NCA 1503, 10, 240, ATCC 12016, RS93 and B. caldolyticus all had similar $\% \mathrm{G}+\mathrm{C}$ contents and showed relatively high levels of DNA hybridization with strain NCA 1503. The implications of these results must be treated with caution since the extent of reassociation was only examined against [ ${ }^{32}$ P]DNA from four organisms.

Characterization of the strains according to antibiotic sensitivity (Table 2) was not possible since the differences in the sizes of the inhibitory zones were small. Bacillus stearothermophilus 136 and strain RS93 showed some similarities, having a slightly greater resistance to several antibiotics, including chloramphenicol, erythromycin, fusidic acid, furazolidone, ampicillin, cloxacillin and penicillin $\mathrm{G}$ than the other strains examined.

Characterization by phage typing (Table 3) was of limited value although the caldo-active strains were similar. Bacillus caldolyticus showed a slightly lower level of infection, perhaps due to the presence of the restriction enzyme BclI (Bingham et al., 1978). Bacteriocin typing may become an important system for distinguishing groups of strains. Thermocin 93 clearly distinguished B. stearothermophilus 136 and B. coagulans from the other strains.

API-ZYM test strips showed the caldo-active strains to be similar, with relatively high levels of extracellular enzyme production (Table 4). Bacillus stearothermophilus 262 showed very limited activity and strains NCA 1503 and 10 did not show any overall close similarity.

Our study indicated that taxonomic grouping based on genotype data and phenotypic characterization did not always coincide. Six of the strains examined remained in the same groups when characterized on several different criteria, i.e. Walker \& Wolf's (1971) classi- 


\section{Table 11. Classification of strains based on matching coefficient}

Matching coefficient (Ss) was calculated from $\mathrm{Ss}=(a+d) /(a+b+c+d)$, where $a$ is the number of characters positive in both strains, $b$ is the number of characters positive in strain 1 and negative in strain $2, c$ is the number of characters negative in strain 1 and positive in strain 2 and $d$ is the number of characters negative in both strains. The 43 characters used for the calculation were all the tests from Table 1, with growth in $\mathrm{NaCl}$ recorded at $2 \%$ and $5 \%$ and starch hydrolysis recorded as $\mathrm{R}$ (restricted), positive or negative, and susceptibilities to phages TP1C, TP84 and 12016b and to thermocin 93 from Table 3.

\begin{tabular}{|c|c|c|c|c|c|c|c|c|c|c|}
\hline Strain & $\begin{array}{c}\text { NCA } \\
1503\end{array}$ & 10 & 240 & $\begin{array}{l}\text { B. caldo- } \\
\text { lyticus }\end{array}$ & $\begin{array}{r}\text { ATCC } \\
12016\end{array}$ & $\begin{array}{l}\text { B. caldo } \\
\text { tenax }\end{array}$ & $\begin{array}{l}\text { B. caldo- } \\
\text { velox }\end{array}$ & 262 & RS93 & 136 \\
\hline NCA 1503 & $100 \%$ & & & & & & & & & \\
\hline 10 & 91 & $100 \%$ & & & & & & & & \\
\hline 240 & 88 & 80 & $100 \%$ & & & & & & & \\
\hline B. caldolyticus & 84 & 80 & 86 & $100 \%$ & & & & & & \\
\hline ATCC 12016 & 65 & 70 & 63 & 67 & $100 \%$ & & & & & \\
\hline B. caldotenax & 74 & 72 & 72 & 88 & 68 & $100 \%$ & & & & \\
\hline B. caldovelox & 76 & 74 & 74 & 91 & 70 & 98 & $100 \%$ & & & \\
\hline 262 & 77 & 70 & 88 & 77 & 65 & 76 & $74^{\circ}$ & $100 \%$ & & \\
\hline RS93 & 63 & 53 & 70 & 65 & 60 & 65 & 67 & $60^{\circ}$ & $100 \%$ & \\
\hline 136 & 49 & 44 & 56 & 60 & 53 & 60 & 63 & 51 & $80^{\circ}$ & $100 \%$ \\
\hline
\end{tabular}

\begin{tabular}{|c|c|c|c|}
\hline $\begin{array}{l}\text { Matching } \\
\text { coefficient }\end{array}$ & Group 1 & Group 2 & Group 3 \\
\hline $90-99 \%$ & $\begin{array}{l}\text { B. caldotenax } \\
\text { B. caldovelox }\end{array}$ & & $\begin{array}{l}\text { B. stearothermophilus } \\
\text { NCA } 1503 \\
\text { B. stearothermophilus } 10\end{array}$ \\
\hline $80-89 \%$ & B. caldolyticus & $\begin{array}{l}\text { B. stearothermophilus } 136 \\
\text { B. stearothermophilus } \mathrm{RS} 93\end{array}$ & $\begin{array}{l}\text { B. stearothermophilus } 240 \\
\text { B. caldolyticus }\end{array}$ \\
\hline $\begin{array}{l}70-79 \% \\
60-69 \%\end{array}$ & $\begin{array}{l}\text { B. stearothermophilus } 262 \\
\text { B. stearothermophilus } \\
\text { ATCC } 12016\end{array}$ & & $\begin{array}{l}\text { B. stearothermophilus } 262 \\
\text { B. stearothermophilus } \\
\text { ATCC } 12016\end{array}$ \\
\hline
\end{tabular}

fication, esterase analysis, matching coefficients and genotype data. However, $B$. caldolyticus and B. stearothermophilus 262 showed features common to groups 1 and 3. Bacillus stearothermophilus ATCC 12016, while closely fitting Walker \& Wolf's group 1, showed a \% $\mathrm{G}+\mathrm{C}$ content similar to group 3 but had an overall similarity of less than $70 \%$ with either group. Strain RS93, while appearing phenotypically closely related to strain 136 , showed a very different genotype.

Bacillus caldolyticus appeared from our study to be a strain of B. stearothermophilus, with a $\% \mathrm{G}+\mathrm{C}$ content falling within the upper group for $B$. stearothermophilus. Bacillus caldotenax and $B$. caldovelox were almost identical to each other and probably represent closely related strains of the same species. They have a $\% \mathrm{G}+\mathrm{C}$ content of approximately $65 \%$, higher than that previously found in B. stearothermophilus. Strain 262 also showed this high $\% \mathrm{G}+\mathrm{C}$ content and emerged as a related strain. Bacillus stearothermophilus 136 , while showing close phenotypic similarities with strain RS93, had a genotype very similar to that of B. coagulans.

\section{REFERENCES}

Atrinson, A. (1973). The purification of microbial enzymes. Process Biochemistry 8 (8), 9-12.

Atkinson, A., Evans, C. G. T. \& Yeo, R. G. (1975). Behaviour of Bacillus stearothermophilus grown in different media. Journal of Applied Bacteriology 38, 301-304.
Baillie, A. \& Norris, J. R. (1963). Studies on enzyme changes during sporulation in Bacillus cereus using starch gel electrophoresis. Journal of Applied Bacteriology 26, 102-108.

Baillie, A. \& Walker, P. D. (1968). Enzymes of thermophilic aerobic spore-forming bacteria. Journal of Applied Bacteriology 31, 114-119. 
Bingham, A. H. A., Atkinson, A., Sciaky, D. \& Roberts, R. (1978). A specific endonuclease from Bacillus caldolyticus. Nucleic Acids Research 5, 3457-3467.

Brock, T. D. \& Freeze, H. (1969). Thermus aquaticus, a non-sporulating extreme thermophile. Journal of Bacteriology 98, 289-297.

Buchanan, R. E. \& Gibbons, N. E. (editors) (1974). Bergey's Manual of Determinative Bacteriology, 8th edn. Baltimore: Williams \& Wilkins.

Cazzulo, J. J., Sundaram, T. K. \& Kornberg, H. L. (1969). Regulation of pyruvate carboxylase formation from the apo-enzyme and biotin in a thermophilic bacillus. Nature, London 223, 1137 1140 .

Cazzulo, J. J., Sundaram, T.K. \& Kornberg, H. L. (1970). Mechanism of pyruvate carboxylase formation from the apo-enzyme and biotin in a thermophilic bacillus. Nature, London 227, 11031105 .

COWAN, S. T. \& STEEL, K. J. (1974). Manual for the Identification of Medical Bacteria, 2nd edn. Cambridge: Cambridge University Press.

DenhaRdT, D. T. (1966). A membrane filter technique for the detection of complementary DNA. Biochemical and Biophysical Research Communications 23, 641-652.

Epstein, I. \& Grossowicz, N. J. (1969). Prototrophic thermophilic bacillus: isolation, properties and kinetics of growth. Journal of Bacteriology 99, 414-417.

Heinen, W. (1971). Growth conditions and temperature-dependent substrate specificity of two extremely thermophilic bacteria. Archiv für Mikrobiologie 76, 2-17.

HeINEN, U. J. \& HEINEN, W. (1972). Characteristics and properties of a caldo-active bacterium producing extracellular enzymes and two related strains. Archiv für Mikrobiologie 82, 1-23.

Jung, L., Jost, R., Stoll, E. \& ZuBer, H. (1974). Metabolic differences in B. stearothermophilus grown at $55^{\circ} \mathrm{C}$ and $37^{\circ} \mathrm{C}$. Archives of Microbiology 95, 125-138.

Marmur, J. (1961). A procedure for the isolation of deoxyribonucleic acid from microorganisms. Journal of Molecular Biology 3, 208-220.

Marmur, J. \& Doty, P. (1962). Determination of the base composition of deoxyribonucleic acid from its thermal denaturation temperature. Journal of Molecular Biology 5, 109-118.

Sargeant, K., EAST, D. N., Whitaker, A. R. \& Elsworth, R. (1971). Production of Bacillus stearothermophilus NCA 1503 for glyceraldehyde3-phosphate dehydrogenase. Journal of General Microbiology 65, iii.

Shat, H. N., Williams, R. A. D., Bowden, G. H. \& Hardie, J. M. (1976). Comparison of the biochemical properties of Bacteroides melaninogenicus from human dental plaque and other sites. Journal of Applied Bacteriology 41, 473-492.

Sharp, R. J., Comer, M. J., Bingham, A. H. A. \& AtKinson, A. (1979). Partial characterization of a bacteriocin (thermocin) from Bacillus stearothermophilus RS93. Journal of General Microbiology 111, 449-451.

Singleton, R. \& Amelunxen, R. E. (1973). Proteins from thermophilic microorganisms. Bacteriological Reviews 37, 320-342.

WALKER, P. D. \& WoLF, J. (1971). The taxonomy of Bacillus stearothermophilus. In Spore Research, pp. 247-264. Edited by A. N. Barker, G. W. Gould \& J. Wolf. London: Academic Press.

Williams, R. A. D. (1975). Caldoactive and thermophilic bacteria and their thermostable proteins. Science Progress 62, 373-393. 